\title{
Interactive Collaboration for Virtual Reality Systems related to Medical Education and Training
}

\author{
B.R.A. Sales, L.S. Machado \\ Department of Informatics of Federal University of Paraíba, Paraíba, Brazil \\ R.M. Moraes \\ Department of Statistics of Federal University of Paraíba, Paraíba, Brazil
}

\begin{abstract}
Virtual Reality (VR) systems have a recent story from the social and practical applications point of view. The main idea related to this area refers to the use of three-dimensional environments in which users can explore and interact in virtual worlds and feel immersed and involved in this process. Nowadays the state of art in VR researches includes collaborative VR environments for medical training and teaching. The use of such collaborative environments allows students to share their knowledge or to be assisted by a tutor during a simulation. This paper presents the development process of a module for interactive collaboration to CyberMed, a framework available since 2004 to speed up the building of medical simulations based on VR technologies. The module for collaboration that was integrated to this framework was used to develop test applications.
\end{abstract}

\section{INTRODUCTION}

Virtual Reality (VR) has been established as an area that provides an effective and motivating way to help teaching in several fields (Kim \& Park 2006, Riva 2003). It allows multi-sensory experiments by user interaction with virtual environments (VE) generated by computer. In the medical area, VR applications have become an alternative tool for training of medical procedures and a tool to support the implementation of laboratory practices.

Some factors like the cost of training, as well as ethical issues, have contributed to the search of new training methods of medical procedures. In this context, VR systems can simulate and also improve the traditional training methods. Thus, the use of VE permits the student to interact and improve their skills in environments that simulate real activities. The environments may be developed by focusing on key-points of a procedure or even points at which medical errors are often noticed. Moreover, there is the possibility for repeat the procedure as many as desired, until the student is able to perform it on a real situation. However, there are procedures that are performed not only by one person, but by a team of professionals together. In these cases, it is necessary to add collaborative features in the VE in order to allow simulating real-world situations. Thus, it becomes possible interaction of multiple participants in what is called Collaborative Virtual Environment (CVE) (Benford et al. 2001).
In this work the collaboration in Virtual Environments is discussed as a way to help students and professionals in the training of medical procedures, independently of their geographical location. The paper describes the design and development of a Collaboration module to be integrated into the framework CyberMed. The framework integrated with the module was used to design a case study on a collaborative virtual environment for bone marrow harvest.

\section{COLLABORATION FOR EDUCATIONAL VIRTUAL ENVIRONMENTS}

The collaboration concept has various definitions in literature. In this work, collaboration is considered as the information exchange by user interaction in a shared environment, i.e., there is collaboration when two or more users are included in a shared space performing some task together.

Virtual Environments have been used in educational context in several areas of knowledge (Youngblut 1998), including medicine (Riva 2003). In general, these environments are designed to allow the acquisition of specific knowledge through the use of computer applications.

The educational VEs can be explored to develop an environment that allows the presence of multiple users. Therefore, it is necessary to use techniques to allow people in different locations feel immersed in a common environment (Singhal \& Zyda 1999). 
Thus, the use of network VE makes possible to replicate situations in which participants work together for a common goal. In this context, the participants of such environments can be able to learn and, at the same time, contribute for other users learning. The use of such environments in medicine context brings benefits to student learning. For example, collaborative simulations can be used to overcome distance problems and provide remotely monitored training in regions without specialists. In this case, a specialist can perform techniques through simulations or interactions in the VE and users can experience and follow the procedure. Furthermore, collaboration in VR environments also enables students to be a part of simulations with others situated in distant locations, promoting the exchange of knowledge.

\section{VIRTUAL REALITY IN MEDICINE}

As a flexible tool, Virtual Environments have been designed to simulate several real world problems and used with different purposes, approaches or objectives. In medicine, VR can be applied joined to other technologies to help students in the learning process. Applications of human structures three-dimensional (3D) visualization and simulations of medical procedures have been analyzed by researcher groups as tools for teaching and training.

The study of anatomy, as example, is often made by static image visualization. In this context, the VR tools can be used to generate $3 \mathrm{D}$ visualization of anatomy structures. Thus students are able to interact with these structures and visualize them from different points of view. Moreover, the Virtual Environments are a way to replace some methods that are frequently questioned about financial and ethical issues, as the use of human corpse or live animals.

The simulations of medical procedures enable users to perceive sensorial cues, into a VE, similarly to real procedures. This is important to prepare students technically and psychologically to deal with real situations. The current VR medical simulations provide high resolution graphics, haptic interaction and a diversity of functionalities that improve the training efficacy (Sung et al. 2003, Friedl et al. 2002).

\subsection{Collaborative Virtual Environments for Medical Training}

Dev (2002) developed two simulation-based learning environments, one for teaching anatomy and other for practicing basic surgical maneuvers like probing, cutting, and suturing. The first environment contains a three-dimensional model of the hand which has interactive rotating view. The hand can be viewed in stereoscopic visualization at different depths of dissection. The second environment contains models of pelvic anatomy and surgical tools to enable the medical training, even collaboratively. Figure 1 illustrates the two learning environments developed. The left image (Fig. 1a) shows the 3D hand model used by the anatomical learning environment. The others two images (Fig. 1b) show the virtual environment for practicing basic surgical maneuvers.

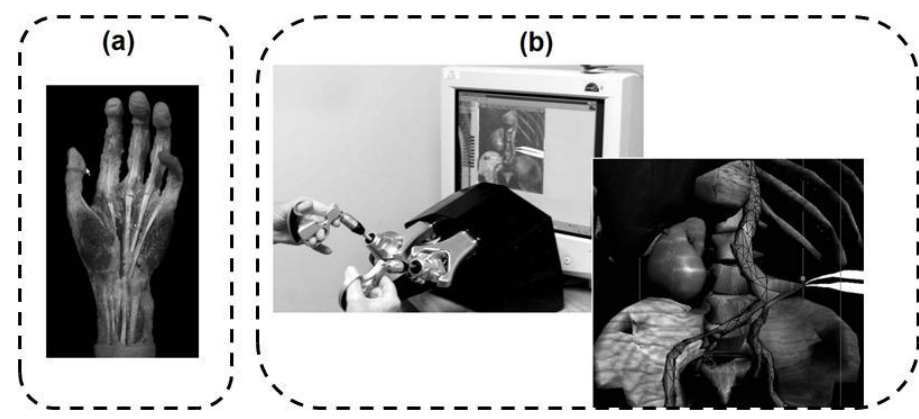

Figure 1. Learning environments developed by Dev (2002).

Another use of Collaborative Virtual Environments in medicine is the remotely conduction of classes through haptic interaction. In this case, an instructor performs a task while students follow the movements remotely. Gunn (2005) built an environment to simulate the gall bladder removal with instructor and student working in the same virtual space on a 3D model of body organs (Fig. 2).

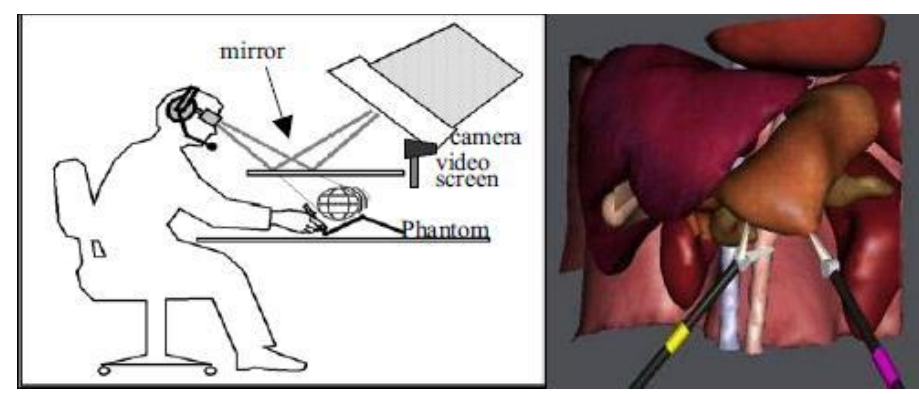

Figure 2. Collaborative virtual environment to conduct a remote surgical master class using haptic device (Gunn et al., 2005).

There are several virtual environments that provide alternative ways to perform medical training. However, the development of these applications requests a lot of time and hard work. Frequently, a framework is used to support the development of medical training virtual environments.

\subsection{Frameworks for medical training simulations development}

A framework is an abstract project and implementation that provides a set of services to be used in application development (Bosh \& Mattsson 1999). Frameworks allow decreasing development time and offer several functionalities that can be selected by the programmer, allowing the reuse of components. 
The tendency to reuse services encourages researcher groups to spend their time on frameworks development. To support the development of medical training virtual environments, the frameworks have to deal with some requirements, such as: different visualization modes, complex modeling, haptic feedback, etc.

Recently, some solutions were proposed and developed to help the design and implementation of simulators for medicine. Examples include frameworks as SOFA (Allard et al. 2007), ViMeT (Oliveira et al. 2006), Spring (Montgomery et al. 2002), GiPSi (Goktekin et al. 2004). Although the same goal, they do not include a tool that assess the simulation performed in the applications developed with them. Furthermore, to allow the representation of scenarios in which there are two or more people working together, the frameworks have to provide collaboration functionalities. Some of these frameworks provide features to create simulations with collaborative tasks. Nevertheless, they generally allow collaboration activities in only one or few modes. Some aspects like collaborative object manipulation and remote mentoring were not addressed yet.

\section{CONCEPTION}

The development of a collaboration module to be integrated to the CyberMed framework came up as purpose of framework functionalities increase. It was intended to enhance the framework power by providing activities related to collaboration among users. This module aims to provide multi-user collaboration to medical simulation VEs in several ways, such as two different object manipulation modes, interaction devices, etc. The module was designed to let the developers free to choose what way would best fit into their specific case. The collaboration module, called CybCollaboration, is a set of services at a high level that deals with network communication and devices requirements. In this way, the user need to be focused only on the specific characteristics of the desired collaboration scenario.

CyberMed was chosen as the framework to contain the collaboration module because it is a free and stable development tool with a large diversity of features compared to others with similar purposes (Machado et al. 2009). CyberMed contains packages for visualization, collision, deformation, assessment, support for haptic and tracker devices, etc. The CybCollaboration module aims to enable all the features already available on CyberMed and also the building collaborative medical simulations.

The research related to this work aims to examine ways in which users could collaborate in medicine VEs. To improve the usability of the module, it was decided to support collaboration across conventional and unconventional devices such as mouse, haptic and tracker devices.

\section{DEVELOPMENT}

This section describes the development of the CybCollaboration module. Initially, requirements analysis was done to elucidate the needs of the module. The development was divided in three parts: architecture definitions, CyberMed modifications and; CybCollaboration design and implementation.

\subsection{Architecture}

The CybCollaboration has direct dependency on CybNetwork, which consists in a set of classes implemented in $\mathrm{C}++$ using sockets, designed to be the CyberMed communication module. The CybNetwork was developed and tested, but had not yet been integrated into CyberMed architecture. To integrate these two modules to the framework, it was done changes in the CyberMed Application Engine layer. This layer provides services that are used directly by the CyberMed user, such as visualization, collision, deformation, assessment and haptic interaction. Figure 3 allows the observation of the CyberMed architecture after the insertion of CybCollaboration and CybCommunication modules.

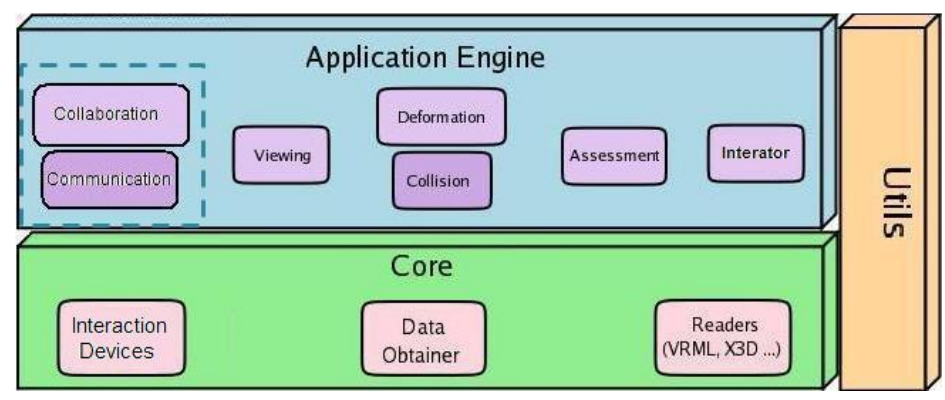

Figure 3: General architecture of CyberMed with communication and collaboration modules (Machado et al. 2009).

\subsection{CyberMed modifications}

The integration of CybCollaboration module forced some changes on CyberMed modules. During a collaborative simulation, avatars (devices representations on VE) of remote users must be presented in the environment together with the local user avatar, in a way that every user can realize the presence of the others. Since this multiple avatar presentation was not possible in CyberMed, it was necessary to reformulate the structure of some Interator Management classes.

Some changes were made on CybView (visualization module) and also on CybCollision (module that treats with collision events). They were adapted to deal with multiple users' avatars. 


\subsection{Design and Implementation}

The design of CybCollaboration module aimed at simplifies the development of collaborative applications. Moreover, it is also possible to the user the implementation of new collaboration methods and the addition of these methods in CyberMed.

The class diagram shown in Figure 4 is a simplified illustration of how the CybCollaboration module is defined. The main class, also named CybCollaboration, contains generic operations common to all collaboration methods. The other classes were divided according to the device type used in collaboration. The main properties of collaboration are stored in class CybCollaborationProperties. It also contains operations to choose the type of desired object manipulation at the collaboration scenario. As a particular behavior, the class CybAssistedHapticCollab is sponsor for assisted haptic collaboration, i.e. a mode of collaboration in which the participants are guided by a tutor during a task.

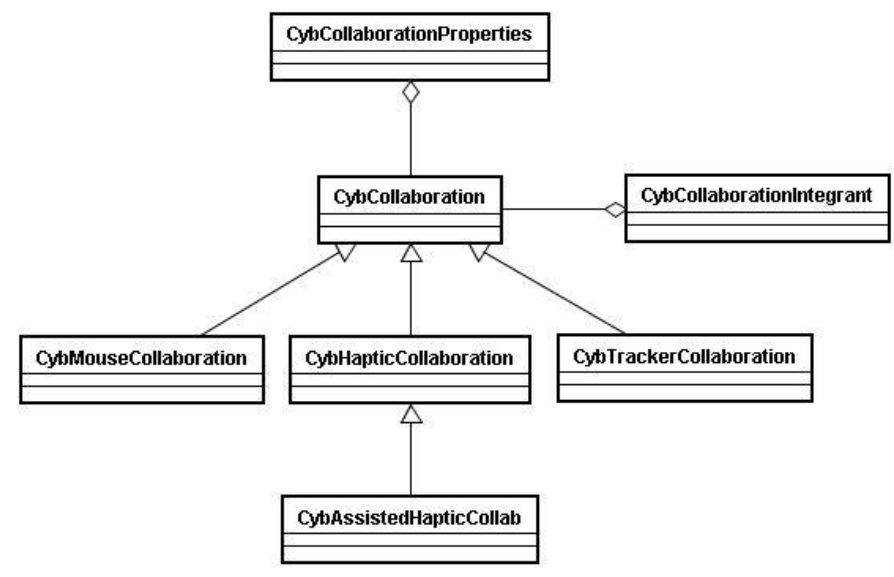

Figure 4: General class diagram of CyberMed collaboration module.

The implemented classes have increased $\mathrm{Cy}$ berMed framework with a set of features related to collaborative activities. The main features referred to:

Number of users: methods to support collaboration with different numbers of users (one-to-one, one-to-many, many-to-many);

Object manipulation: there are two different ways implemented to manage the user interaction with objects in the VE. The first one allows users to interact with the same object simultaneously (Fig. 5a). The users' interactions are unified and the result is applied to the object. The other approach, blocks the object for other users, when it already been interacted by a user (Fig. 5b); (a)

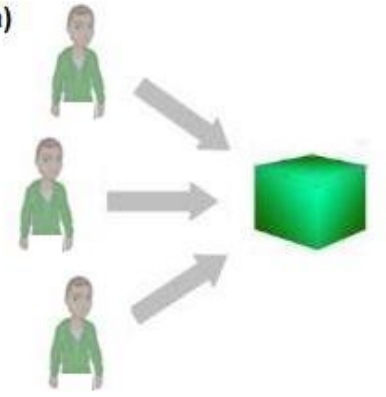

(b)

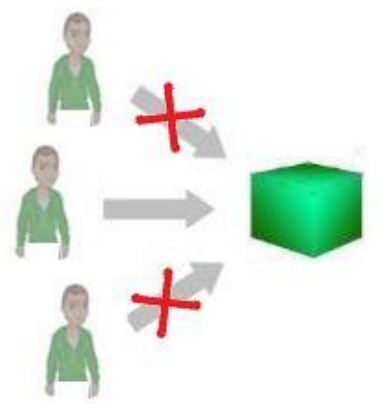

Figure 5: Two different approaches for object manipulation. In (a) the interactions performed by users are applied to the object like a resultant interaction. In (b) only one user is able to interact with the object, while the others have to wait.

Devices: collaboration through the use of conventional devices like mouse, and unconventional, like haptic systems (that provides force feedback), or tracker systems;

Remote Mentoring: determination of a participant to tutor the others within the environment. This mentoring can be done at different levels. With the use of haptic systems, for example, it is possible to determine if users shall be restricted to movements performed by the tutor or if only a visual representation of the tutor device will appear in the user environment to guide him in the activity.

\section{RESULTS}

After the development and integration of the CybCollaboration module, applications were built to test the module. In particular, a simulation of a bone marrow harvest procedure was adapted to provide collaboration with touch and force feedback among remote users. In this application a user was selected as a tutor and guided all other users connected.

The bone marrow harvest consists of a procedure in which the doctor interacts with the patient in order to extract material for donation. During the procedure, the doctor has no visual information from inside the patient. He must touch the patient externally to determine the correct location to insert the needle. Then he must pierce the layers of tissue to reach the interior of the bone where the marrow is collected. Two important things are: the site of needle insertion and the force that the doctor will apply to reach the desired location. The correct site of needle insertion is the iliac bone which contains the correct width and hardness to the procedure realization. If this force is applied in excess, it can be harmful for the patient and may cause sequel (Machado et al. 2002, 2003).

The simulation of bone marrow harvest (Fig. 6) is divided into three modules: Observation, Location and Harvest. In the three modules, users will be able to interact with the system by the use of a haptic de- 
vice (Phantom Omni). The interactions performed by the guide user are perceived in real-time by all users connected to the simulation. Then, they are able to feel sensory impressions and experience the movements and forces presented in the procedure.

In the tests were used two computers connected to a Gigabit LAN. Both computers were equipped with haptic devices and one user was defined as the tutor while the others were tutored, perceiving only the tutor interactions. Two other tests were also conducted with three computers equipped with haptic devices of different models and five computers with interaction through mouse (Fig. 7), respectively. In all experiments, the information send and receive occurred in real-time.

Other experiments performed consisted on the free manipulation of devices by every user present in the virtual environment. The experiments were made with the same number of computers and devices of the previous tests. All participants were able to observe the position of self-avatar as well as the other participants avatars.
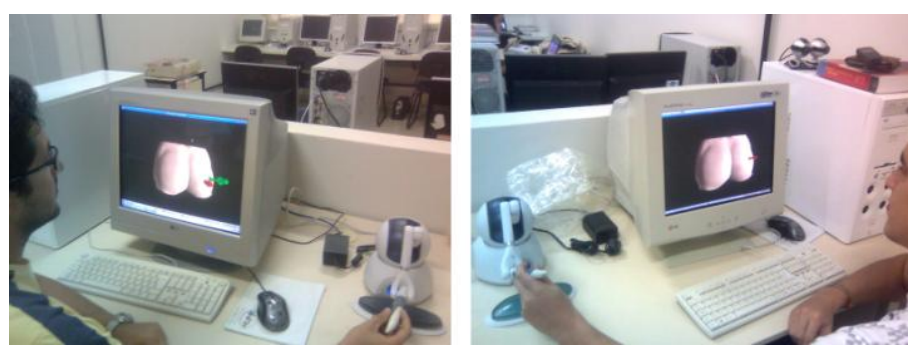

Figure 6: Collaborative simulation of the bone marrow harvest procedure. Haptic device Phantom was used to interact with models.
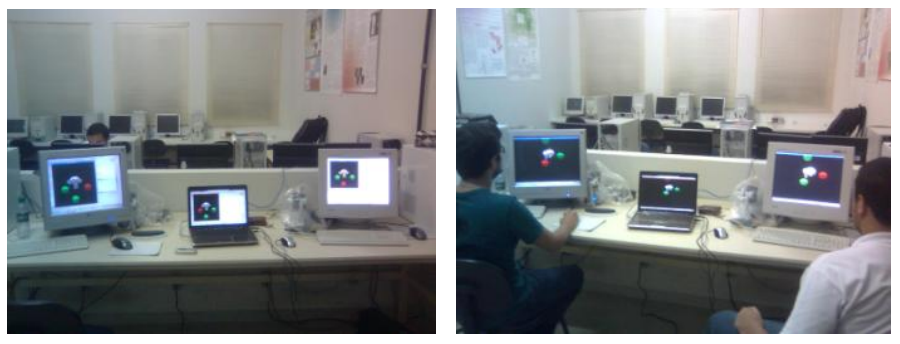

Figure 7: Application to test mouse collaboration scenario.

\section{CONCLUSIONS}

The results pointed out the potential of using collaborative virtual environments for education, particularly in medicine. The possibility of share knowledge in VR supported medical simulations is a promising alternative in medical training field. Moreover, the collaborative simulations enable professionals to demonstrate, through tactile interactions, how students must proceed during a specific medical procedure. This possibility allows students of remote places to increase your medical skills by sharing and receiving knowledge from experts.

In this work it was detailed three applications to test and validate the CybCollaboration module functionalities. Also, a complete simulation of the bone marrow harvest procedure was adapted to be performed collaboratively. In this sense, this research suggests discussions on validation of collaborative applications to medicine. It is also important to highlight that, whereas the use of collaboration for training is not limited to medical field, researches can be conducted to examine how collaboration can be inserted to assist training in other areas.

Future work includes the expansion of the collaboration features to the addition of object manipulation specific techniques, providing means to participants, in addition to handling, also interactively modify objects of the VE. Ruddle and colleagues describe some techniques for manipulating objects in collaborative virtual environments (Ruddle et al., $2002 \mathrm{a}, \mathrm{b})$. It can be analyzed to be added to the collaboration module according to its relevance in medical environments. It is important to mention that the basis for implementation of such expansion is already available in the module presented in this work.

The implemented module is available on version 2.0 of CyberMed (CyberMed 2010). The framework can be obtained freely on the project page on the Internet (http://cybermed.sourceforge.net/).

\section{REFERENCES}

Allard, J.; Cotin, S.; Faure, F.; Bensoussan, P.J.; Poyer, F.; Duriez, C.; et al. 2007. SOFA - an Open Source Framework for Medical Simulation. Medicine Meets Virtual Reality 15:1-6.

Benford, S; Greenhalgh, C.; Rodden, T. \& Pycock, J. 2001. Collaborative virtual environments. Communications of the ACM 44(7):79-85.

Bosh, J., Mattsson, M. 1999. Framework Problems and Experiences. In M. Fayad, R. Johnson, D. Schmidt, Building Application Frameworks: Object-Oriented Foundations of Framework Design: 55-82. New York: John Willey and Sons.

CyberMed. 2010. Online: http://cybermed.sourceforge.net/.

Dev, P.; Montgomery, K.; Senger, S.; Heinrichs, W.L.; Srivastava, S.; Waldron, K. 2002. Simulated Medical Learning Environments on the Internet. Journal of the American Medical Informatics Association 9(5):437-447.

Friedl, R.; Preisack, M.B.; Klas, W.; Rose, T.; Stracke, S.; Quast, K.J.; et al. 2002. Vritual Reality and 3D Visualizations in Heart Surgery Education, Heart Surg Forum 5(3):17-21.

Goktekin, T.; Cavusoglu, M.C.; Tendick, F.; Sastry, S. 2004. GiPSi: An Open Source/Open Architecture Software Development Framework for Surgical Simulation. In Proceed- 
ings of the International Symposium on Medical Simulation, Cambridge.

Gunn, C.; Hutchins, M.; Stevenson, D.; Adcock, M.; Youngblood, P. 2005. Using collaborative haptics in remote surgical training. Eurohaptics Conference and Symposium on Haptic Interfaces for Virtual Environment and Teleoperator Systems (WHC'05), Italy.

Kim, L. \& Park, S.H. 2006. Haptic interaction and volume modeling techniques for realistic dental simulation. The Visual Computer: International Journal of Computer Graphics 22:90-98.

Machado, L.S.; Mello, A.N.; Odone Filho, V.; Zuffo, M.K. 2002. Virtual Reality Simulation of Pediatric Bone Marrow Harvest for Transplant. Medical and Pediatric Oncology. 39(4):282

Machado, L.S.; Moraes, R.M.; Souza, D.F.L. \& Souza, L.C.; Cunha, I.L.L. 2009. A Framework for Development of Virtual Reality-Based Training Simulators. Studies in Health Technology and Informatics 142:174-176.

Machado, L.S; Zuffo, M.K. 2003. Development and Evaluation of a Simulator of Invasive Procedures in Pediatric Bone Marrow Transplant. Studies In Health Technology And Informatics. 94:193 - 195 .

Montgomery, K. et. al. 2002. Spring: A general framework for collaborative, real-time surgical simulation, Proceedings of Medicine Meets Virtual Reality, IOS Press, p. 23-26.

Oliveira, A.C.M.T.G.; Botega, L.C.; Pavarini, L.; Rossatto, D.J.; Nunes, F.L.S.; Bezerra, A. 2006. Virtual Reality Framework for Medical Training: Implementation of a deformation class using Java. In Proceedings of the SIGGRAPH International Conference on Virtual-Reality Continuum and its Applications in Industry (SIGGRAPH '06): 347-351, Hong Kong.

Riva, G. 2003. Applications of Virtual Environments in Medicine. Methods of Information in Medicine 42(5):524-534.

Ruddle, R.A.; Savage, J.C.D.; Jones, D.M. 2002a. Implementing flexible rules of interaction for object manipulation in cluttered virtual environments. In Proceedings of the ACM Symposium on Virtual Reality Software and Technology (VRST'02): 89-96.

Ruddle, R.A.; Savage, J.C.D.; Jones, D.M. 2002b. Symmetric and asymmetric action integration during cooperative object manipulation in virtual environments. ACM Transactions on Computer-Human Interaction 9(6):285-308.

Singhal, S. \& Zyda, M. 1999. Networked virtual environments: design and implementation, New York: ACM Press/Addison-Wesley Publishing Co.

Sung, W.H.; Fung, C.P.; Chen, A.C.; Yuan, C.C.; Ng, H.T.; Doong, J.L. 2003. The assessment of stability and reliability of a virtual reality-based laparoscopic gynecology simulation system. Eur J Ginaecol Oncol. 24(2):143-146.
Youngblut, C. 1998. Educational Uses of V R Technology. Technical Report IDA Document D-2128, Institute for Defense Analyses, Alexandria. 